\title{
Mail Art: La comunicación postal y su devenir en medios masivos
}

\section{Irene Covaleda Vicente}

Universidad de Zaragoza, Campus de Teruel, irenecovaleda87@hotmail.com

\begin{abstract}
Resumen
Los proyectos que se interpelan al uso del espacio público y que abordan las relaciones existentes entre dos ámbitos cuyos límites resultan cada día más difíciles de precisar: lo público y lo privado, son los motivos que usamos para evidenciar el transvase de una a otra esfera.

El Mail Art, por usar el medio del correo, cuyo reparto requiere una tecnología humana, es el que más interesa a este proyecto, que basa la experiencia en el proceso de creación de las postales, cintas, dibujos y su posterior escritura y envío. El Umbral es el icono de las viviendas que representa el límite entre la figura de espectador, y emisor de las piezas que se van a presentar.
\end{abstract}

Todo esto se va a conjugar en un discurso de manera que la película del director M. Haneke, Caché, hile las condiciones con las que el protagonista del film, un personaje acomodado en el centro de París y que goza de un alto estatus, observa, como el medio de comunicación masiva, medio en el que trabaja, se puede convertir en una exposición sobre sí mismo que puede tornarse peligrosa para él y su familia.

A través del análisis de los medios que se van a trabajar en este proceso, se dejarán los límites en un estado desdibujado, de difuso reconocimiento. El espacio construido con la ciudad como principal modelo de la vida comunitaria, es un espacio para ser ocupado, para llenar y vaciar con la presencia real o simbólica y, para interactuar con otras personas. Las reglas y convenciones son entonces, impuestos que se pretenden tambalear con la lectura que propone Mail Art. La comunicación postal y su devenir en medios masivos.

Palabras clave: Mail Art, Comunicación, Espacios públicos y privados, Umbral. 


\section{Introducción}

Son las relaciones existentes entre lo público y lo privado, lo que ha generado la necesidad de elaborar un discurso que plantea la imbricación entre dos corrientes artísticas como son el Mail Art y el Cine.

¿Cómo se imbrican lo público y lo privado?, ¿Cómo se produce la intromisión de los objetivos privados de por ejemplo, los empresarios que dominan los medios de comunicación en nuestra vida privada? Y, ¿de qué manera múltiples asuntos que formban parte del mundo privado (la condicion de género, la indentidad étnica, las preferencias sexuales...etc.) ahora ganan visibilidad pública? Y por el contrario, ¿Cuales son los mecanismos por los que los espacios públicos de nuestras ciudades desaparecen o se deterioran en beneficio de los intereses privados?.

Por un lado, se pretende presentar las motivaciones que han llevado a los autores que conformaron el movimiento artístico Mail Art, a la producción de sus piezas, y por otro, se quiere evidenciar la débil frontera que separan las esferas públicas y privadas a través de un medio de reparto que, con cierta facilidad penetra en la intimidad de los moradores.

El propósito de realizar un análisis desde la perspectiva fílmica es estudiar las reacciones que promueve el cineasta, Michael Haneke al presentar la figura del espectador. Mirar desde un prisma cinematográfico, las formas de resistencia ante una mensajería como la que se recrea en Caché, y las variedades que ofrecen los medios de comunicación, hace posible que se planteen las vías entre los modos de creación que se realizan desde el Mail Art y llevarlo hasta la pantalla de cine.

\section{Mail Art: Comunicción postal}

La sociedad se transforma desde hace unas décadas de forma acelerada, los medios de comunicación, que dan buena cuenta de ello, permiten que se sumen posibilidades de producción y visibilidad, aunque la división entre espacios privados y comunes es necesaria y parece presente en el ánimo social.

Es a mediados de los años sesenta cuando el Mail Art se presenta como una tendencia entre los artistas. Se inicia un interés por formar parte de la red, y este canal introduce la intervención de los espacios, además de ser portador de un mensaje que, en muchas ocasiones establece interacción entre varios individuos. Permite la expresión libre y la circulación de multitud de documentos, ideas y materiales que se conjugan. 
El acceso a los medios y estructuras de información artística, es una de las causas de la proliferación de movimientos como el Mail Art, el video-arte, acciones callejeras y performances, movimientos que apuestan por la integración de la actividad artística en las estructuras de los medios y códigos cotidianos de comunicación, entre otros, a través del correo.

Los tiempos que determinan el presente y el pasado, quedan en permanente revisión y siempre en relación a la inestabilidad que propone el sistema de información.

El umbral que define metafóriamente la división entre la público y lo privado y, es el icono que acompaña al estudio. Las imágenes que se han utilizado en películas como la de Caché, o en La carretera de David Lynch, para definir la cercanía entre estos entornos, el umbral, es articulador de las distintas respuestas que integra tiempo, tensión, y permite establecer relaciones entre aspectos como el espacio.

Los medios de comunicación han convertido los ámbitos cercanos a lo privado en algo absolutamente permeable y casi imperceptible. Los autores que han trabajado con el medio del correo como sistema de intercambio de ideas y, para la elaboración de un discurso, fundamentaron las bases de los grupos Fluxus, Dadá, ZAJ y EINA. Estos, planteaban el cuestionamiento crítico de la imagen desde hipótesis en las que el debate intencionadamente, estaba circuscrito a razones conexas con los dispositivos utilizados en este medio y las diferentes plataformas y espacios para formalizar la comunicación artística.

El Mail Art, es desde sus inicios una red de intercambio de ideas que apuesta por el sistema de correo tradicional. Las posibilidades narrativas que ofrece este medio son diversas y los temas que se han tratado van, desde la denuncia política y social, estudios sobre la imagen y el espacio que ocupa, hasta las intervenciones con intencionalidad participativa. Salir de los parámetros establecidos que han enmarcado los circuitos de exposicion en las galerías de arte es tambien, pertinente de señalar puesto que, reivindicar el derecho a la comunicar reflexiones de distintas índoles ideológicas, alejándose de los intereses de comercialización, que siempre está patente en el animo de los mailartistas. Parece que en el caso de Michael Haneke esto sucede tambien. Haneke, no contempla la posibilidad de agradar al espectador, mas bien, señala directamente a las características menos apreciables del ser humano. En concreto, el protagonista de la historia de Caché, es presentado como un personaje de éxito público, asentado en de la ciudad de Paris. Un canal de televisión emite su programa que está basado en la lectura de los libros mas interesantes del panorama actual, sin embargo, alguien pondrá a George, y a todos los espectadores del film, a través de unas sospechosas cintas enviadas por correo, a pensar sobre la experiencia del límite. El exterior, el interior y la fragilidad del espacio existente entre ambos conceptos se mimetizan entre la intimidad personal y el control que ejerce el sistema de comunicación. 
Desdibujar las fronteras entre los medios, y establecer nexos entre esferas de creación, supone plantear una lectura transversal entre los cauces que mas interés causan a la hora de elaborar un discurso, en este caso entre el Cine y el Mail Art. Puesto que conectar sensaciones entre estas, permite integrar la figura de espectador y trabajar con diversas interpretaciones. Tendríamos que incluir pintores, fotográfora, escultores y hasta poetas y músicos o arquitectos para determinar quienes se dedican a la acción del Mail Art. Se especúla con que han deambulado por los circuitos del correo mas de 3.000 activistas, lo cual, nos da una idea de los muchos interrogantes planteados en los convencionalismos y condicionantes del academicismo. Los trabajos que se construyen desde connotaciones de producción de bajo coste, así como la fiabilidad del exitoso viaje, que en sí mismo, presenta una incógnita que resulta difícil de preveer, es una de las diferencias notables que se podría destacar al comparar corrientes como el cine y el Mail y así lo destaca Fco. Javier Collado:

"Frente a la instantaneidad de otros medios de comunicación más modernos, el medio postal se caracteriza por una fuente de fricción material con el engranaje social del transporte" 1

Y este es el poderoso encuentro que se enfoca en el film del austriaco. Haneke, conecta el material que recibe George, el protagonista, a través del correo, y hace posible la narración de un pasado, construyendo un recuerdo como consecuencia de lo invertido en las intenciones del emisor de las cintas. Este trabajo de intentar saber qué espera el espectador, es además de un juego de palablas, una misterio que preocupa a casi cualquier emisor de mensajes, y a muchos artistas.

En uno de los libros que han influido en la elaboración de esta investigación, Welch. 1995. "Eternal Network. A Mail Art Anthology", por varios motivos pero el principal es que, después de estudiar el movimiento del Mail Art como una red de comunicación, o como un diseño artístico que genera un intercambio, se ha comprendido también, otras intenciones que han podido tener los artistas que forman parte del mismo. Me refiero a que cuando Robert Filliou desarrollaba su concepto de los Eternal Network, estaba pensando en la condición humana, más que en una cuestión artística. Este pensamiento acerca de forma destacada, la mirada hacia la pantalla de cine, y a otro de los libros que hetrabajado para sostener un esquema, que queda retratado en la imagen que precede y que en este caso, los

\footnotetext{
1 Martinez Collado, Fco. J.(1990). La paradoja del medio postal en el arte. en Sarmiento, José Antonio, Arte Postal, Ed. Universidad de Castilla La Mancha, Cuenca.

${ }^{2}$ Welch, C. (1995). Eternal Network: A Mail Art Anthology, Ed. Calgary, University of Calgary.
} 
autores de "Antes y después de Auschwitz"33 se plantean, la figura del espectador desde varias perspectivas. Ya en su film In girum nocto et consuminur igni (1978) Guy Debord, representa a una sociedad del consumo despojada de libertad. Las leyes del mercado que son impuestas, someten al humano y organizan su comportamiento. La entrada, el umbral de la vivienda de George, que es imagen protagonista en Caché, no representa el alivio de personaje principal de este film. La extremada vigilancia que presenta Haneke, sostiene que el lenguaje que se desarrolla entre los miembros que forman parte de la Imposible Comunicación, desengaña al espectador de la posibilidad de comprensión.

Eternal Network realiza también un esfuerzo por visibilizar que la comunidad de artistas que forman el movimiento. Estos, no tienen la necesidad de estar en acuerdo con las ideas políticas, sociales o culturales que se presentan en la obras, pero añade que el intercambio de la diversidad de ideas, no restan identidad a otras. Artistas como George Maciunas, Henry Flynt, Yoko Ono, Joseph Beuys, Milan Knizak, Dick Higgins o Lawrence Weiner entre otros, apuntan que lo importante del intercambio no son cuestiones que encierran la técnica, sino el sentido o intención, con el que se realiza la obra. Inclinamos así el pensamiento de las cuestiones raciales, sociales y culturales que muestra Haneke en una cuestión que no representa para estos artistas un impedimento para la creación.

Walter Grarskamp describe las necesidades de entender este espacio como un estado: uno de la condensación y del cruce de funciones, cuya máxima intensidad se sitúa en el centro de la ciudad ${ }^{4}$. El artista nos dejó un mensaje claro, y es que el arte, no puede obviar su contexto, pues éste, le otorga el significado que posee, así mismo no se debe olvidar lo que forma parte del arte público. Esto es la cultura, la arquitectura y la ciudadanía a la que se dirigen.

Una de las cuestiones que se plantea y que es fundamental en las últimas décadas, es la preocupación, de cómo habitamos el espacio, y la estructura centralizada del entorno. Los inicios de estas cuestiones arrancan, en los años setenta, cuando los museos, como institución, que media el espacio legitimador, es cuestionado y poco a poco sustituido por la ciudad, como el nuevo horizonte de creación. El situacionismo, plantea la importancia de deambular por las calles de la ciudad, y además es entendido como la herramienta para realizar críticas sobre los valores funcionales de los espacios públicos y los privados. El arte pasa a ser un compromiso con la sociedad, y en el intervalo desde 1958, cuando los

\footnotetext{
${ }^{3}$ Ferrando G, Pablo; Moral, J. (2016). Antes y después de Auschwitz La cinta Blanca/La cuestión Humana, Shangrila, Santander.

${ }^{4}$ Grarskamp, Walter, Skulptur. Projekte in Münster fue iniciada en 1977 por Klaus Bussmann, y fue desde su origen una colaboración entre la ciudad de Münster y las entidades responsables del Westfällischen Landesmuseum. El principio de selección en torno a proyectos artísticos específicos fue la base de la exposición concebida por Kasper König y Klaus Bussmann en 1987, y ha sido también el criterio de selección de su tercera edición, dirigida en 1997 por Florian Matzner. Ver el texto de Walter Grarskamp Kunst und Stadt, en AAVV, Skulptur. Projekte in Münster 1997, Ostfildern-Ruit, Verlag Gerd Hatje, p. 11, 1997
} 
situacionistas, proclaman la agitación de construir espacios éticos y estéticos, hasta 1968 que obreros, estudiantes y activistas, sacaron a la calle lo mejor de los cuerpos (y fuerzas) de seguridad del Estado. Los artistas trabajan en este intervalo de tiempo en cuestiones de desarrollo, servicios y compromiso.

La ciudad se muestra como un territorio de territorios, en el que el espacio urbano, configura otros espacios que determinan estriados paisajes y regulado por el Estado. Tomar la calle como terreno para el artista, y para desarrollar cuestiones que van en dirección de una reflexión crítica sobre la representación de las esferas y, es lo que caracteriza la propuesta, en relación con la arquitectura y el nomadismo que define el trabajo de algunos de los artistas nombrados.

Para que la idea prepondere en el entorno donde se ha desarrollado, hemos fijado la mirada al igual que el director de cine, analizado en este texto, el lugar concreto que retrata al propio espectador. Confluyen en los planos de la película Caché, ideas que podemos vincular a la historia de la Francia que machacó a los argelinos y la idea de que todo espectador tiene un pasado oculto. En la película que hemos elegido como ejemplo, sobre todo por la figura de un espectador que no conoce al emisor de los mensajes, y que recibe información del exterior, da lugar al replanteamiento del límite que se ocupa de establecer distancias entre el espacio privado o público. Pues George, nuestro presentador afincado en París, recibe una información en el espacio privado de su vivienda, pero que se realiza desde el espacio público, que en la misma calle que llega a su casa. Entiende que este espacio, en principio, creado para el tránsito público, pero sin embargo, tiene un carácter de cierta índole privada. Es al fin y al cabo, nuestro barrio nuestra calle... y el intruso que se afinca, resulta ser objeto de observación y necesariamente, se tendrá que adaptar a las costumbres de la finca, o del órgano al que se quiera referir.

El uso del Mail Art y su devenir en los medios de comunicación masiva queda definida con la reflexión entre la intimidad y de la carga de significado que se presenta a través de los medios, y se suma, la disparidad de la exposición que parece ocupar un espacio cada vez mayor entre los ciudadanos, que además, contrasta con el animo social a la necesidad de considerar un espacio como privado o propio.

La casa es, más aún que el paisaje, un estado del alma. Incluso reproducida en su espacio exterior, dice una intimidad ${ }^{5}$.

La puerta, es lo que delimita los espacios. La frontera, que separa la actitud que retrata al actor según el espacio que ocupe, y que hace de filtro entre la propiedad emocional de este espacio privado y el espacio vivido en el exterior del Umbral $^{6}$.

\footnotetext{
${ }^{5}$ Bachelard, G. (2005). La poética del espacio, Fondo de Cultura Económica, México.
} 
Es oportuno que además de estos detalles que se observan en este inicio a un discurso que conjuga y auna los medios de comunicación, el film Caché y el Mail Art, hacer mención de la intencionalidad que presentan las corrientes al poner al espectador a contemplar la pared que refleja una vida. Éste resulta ser un aforismo de Godard; La representación nos consuela de la tristeza de la vida, y la vida nos consuela de todo lo que la representación no $\mathrm{es}^{7}$.

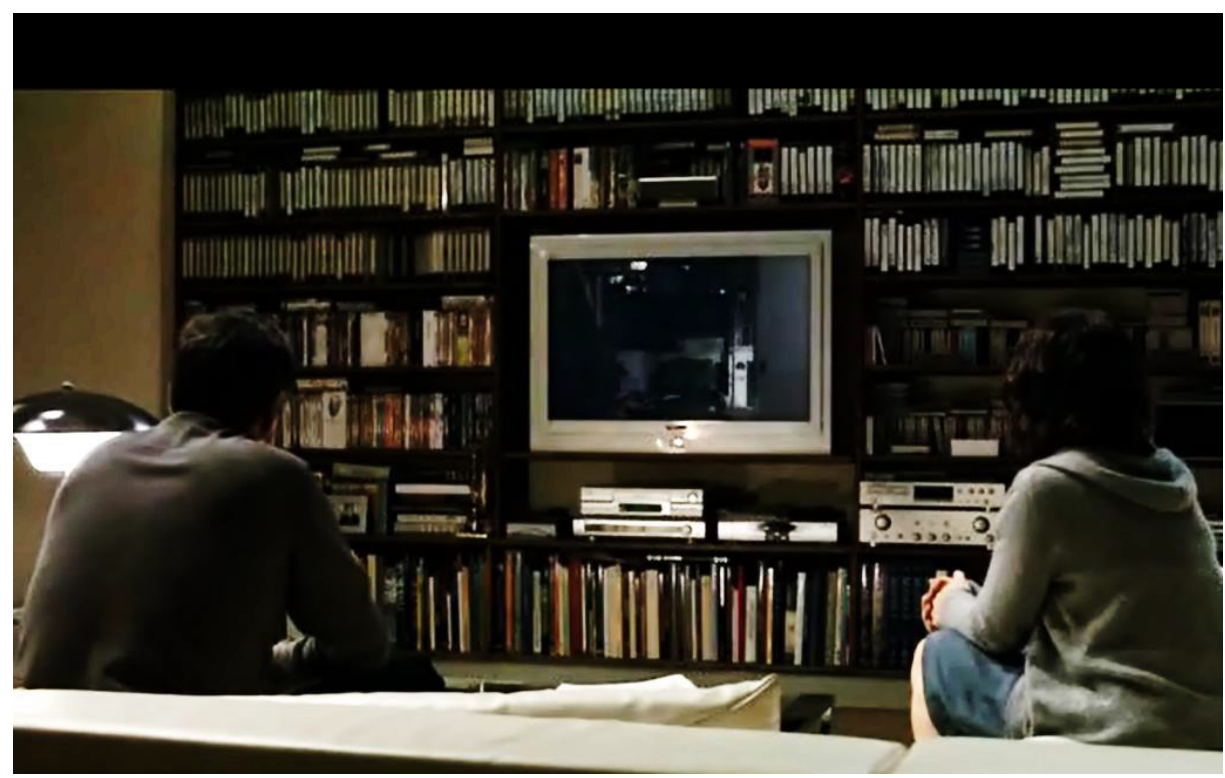

Figura 1: Michael Haneke: Fotograma de Caché (2005), París.

\footnotetext{
${ }^{6}$ Delgado, M.(1999). El animal público, Barcelona.

${ }^{7}$ Recogido del documental producido por el Instituto Nacional de Audiovisual y el Canal + de Francia, dirigido por Michael Royer titulado Godard y la televisión.
} 

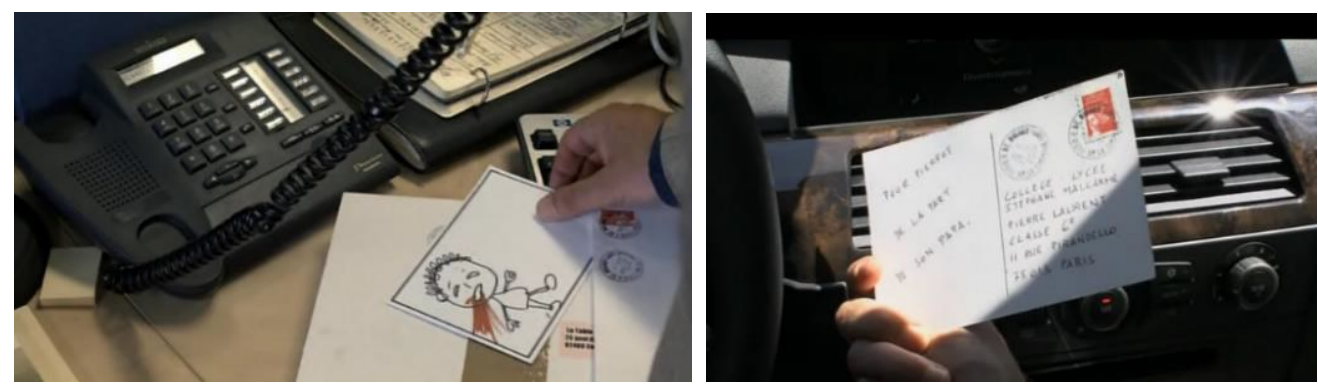

Figura 2: Michael Haneke: Fotograma de Caché (2005), París.

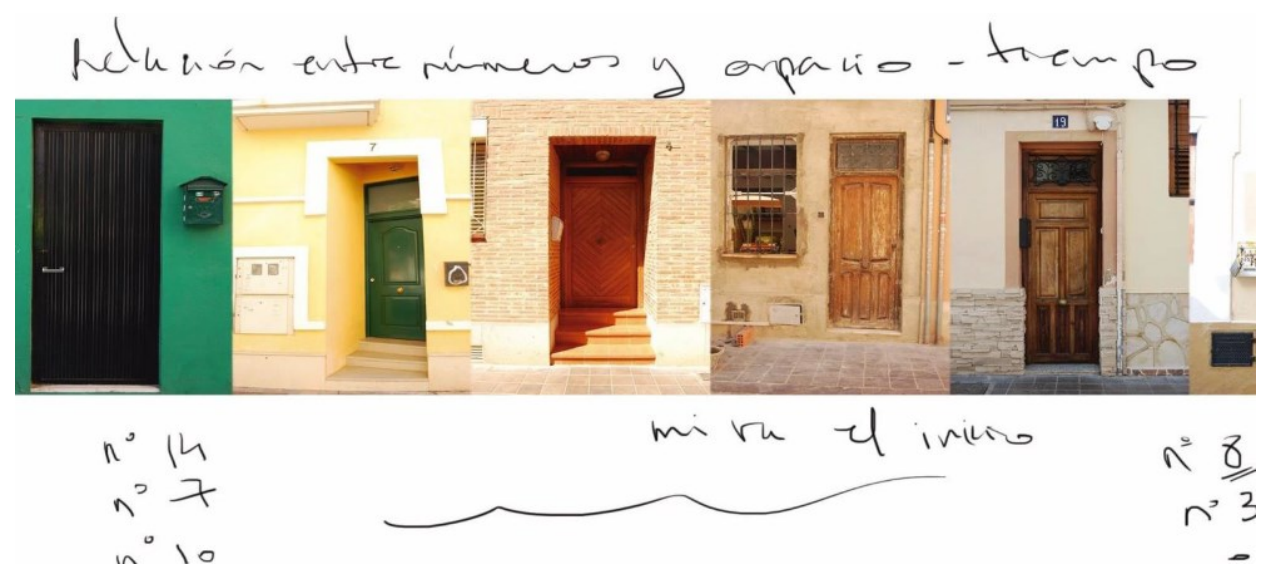

Figura 3: Irene Covaleda. Notes al Peu. Exposición Individual Sala de Exposiciones La Muralla.Enero 2017. 


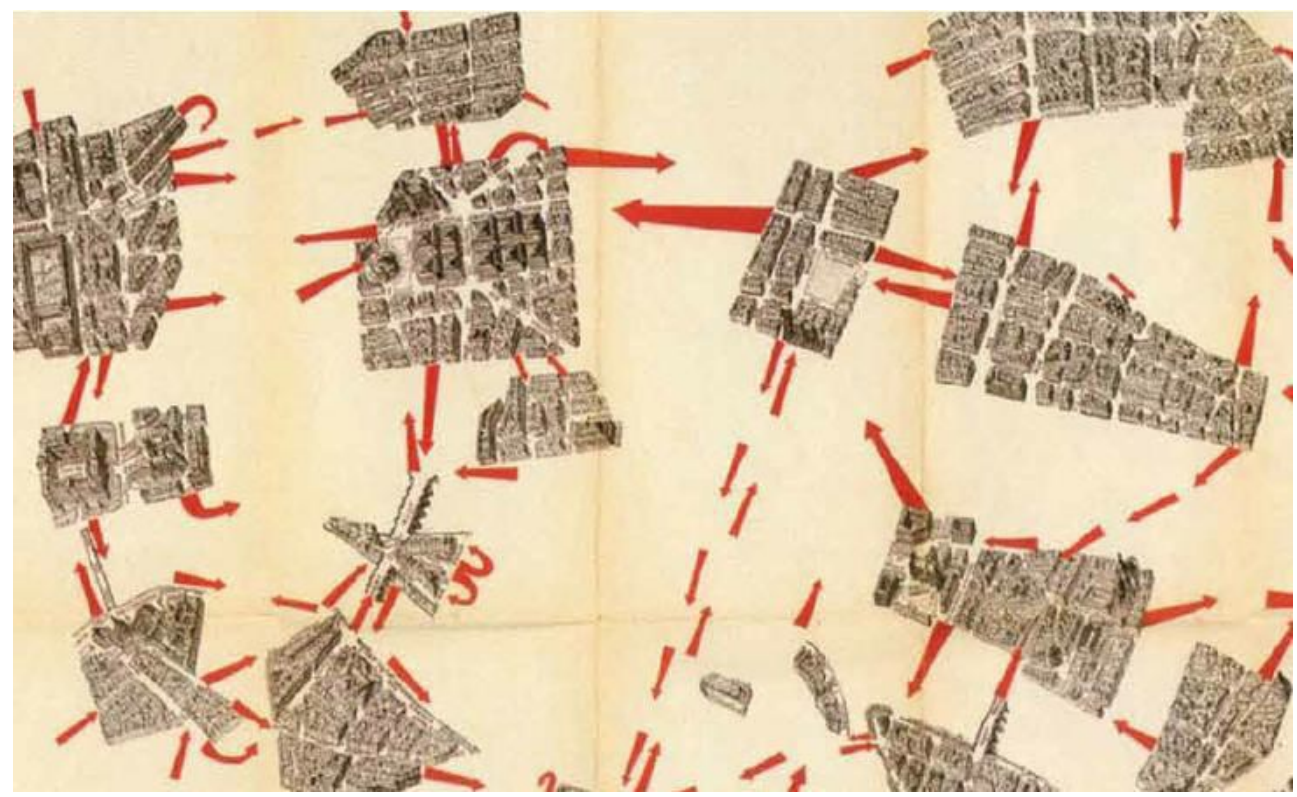

Figura 4: Guy Debord: Psychogeographic guide of Paris, 1957.

\section{Conclusiones}

Añadir, que una vez finalizado este trabajo, desde la memoria inmediata, se considera fundamental para este proceso de trabajo la imbricación de varias disciplinas artisticas, y el espacio bidimensional, la imagen fotográfica y las sendas que hemos construido entre todos los que formamos parte de este proyecto.

Es cierto que el Mail Art, ofrece un amplio abanico de posibilidades a las que estamos deseando acceder y desarrollar otras obras, que se plantean para ser compartidas con personas desconocidas. Analizar el proceso del trabajo y basar los paseos en el movimiento de la deriva, son aspectos que hemos con los que hemos procurado establecer equilibrio, así como con las distintas partes de los procesos artísticos de los que hemos hecho mención. El espacio público que ha sido retratado en una franja de imágenes, que muestran espacios comunes a muchos lugares y nos dan información suficiente de dónde se está generando el trabajo. 


Figures
\begin{tabular}{l|}
\hline Michael Haneke: Fotograma de Caché (2005), París. \\
\hline Michael Haneke: Fotograma de Caché (2005), París. \\
\hline $\begin{array}{l}\text { Irene Covaleda. Titulo de la Exposición: Notes al Peu. Exposición } \\
\text { Individual. Sala de Exposiciones La Muralla. Enero } 2017\end{array}$ \\
\hline Guy Debord: Psychogeographic guide of Paris, 1957. \\
\hline
\end{tabular}

\section{Referencias}

Albright, T.(1990). Correspondence. Rolling Stone, 107, 28-29. Aliaga, Juan Vicente \& Cortés, José Miguel (1990). Arte Conceptual revisado. Valencia.

Anleo, X. (1983). ¿Que es el arte correo?, Revista Tinta China, 24 Agosto Sevilla.

Baroni, V. (1995). Ray Johnson Remembered. Arte Postale!, 69. Recuperado en diciembre de 2002 de http://www.sapienza.it

Baroni, V. (2005). Postcarts: Cartoline d'artista. Roma: Coniglio Editore.

Baroni,V. (2005). Dodo, no Dada: reflexiones de verano sobre un buzón brilloso. En Delgado, Fernando G. \& Romero, Juan C. (Eds.). El arte correo en Argentina, Arte Correo Vórtice, Buenos Aires.

Barreeche, JJ. (1971). Colección SI-NO el cine, Editorial: BRUGUERA, S.A Mora la nueva, Barcelona.

Bazin, A. (2005). ¿Qué es el cine? RIALP, Madrid, 2001 del espacio, Fondo de Cultura Económica, México.

Bachelard, G. (2005). La poética del espacio, Fondo de Cultura Económica, México.

Blasco, Gargallo J. (2016) Lo que siempre queda del arte seminario Visiona: Memoria y desacuerdo: Políticas del archivo, registro y álbum familiar. Huesca.

Bleus, G. (1984). Commonpress 56. Catálogo de exposición. Tienen, Bélgica: Museum Het Toreke,

Bleus, G. (2005). Mail-artcards for Netland (pp.93-105). En Baroni, Vittore (Ed.). Postcarts: Cartoline d'artista. Roma: Coniglio Editore

Bleus, G. (2010). Informe administrativo sobre arte postal. En Sousa, Pere Mail art: la red eterna (pp. 88-90). Sestao: La única puerta a la izquierda - L.U.P.I. / Merz Mail Barcelona.

Bloch, M. (1995). A Ilustrated Introduction to Ray Johnson (1927-1995). Recuperado en mayo de 2011 de http://www.panmodern.com/Ray.html 
Crane, M. (1984). A definition of Correspondence Art. En Crane, Michael \& Stofflet, Mary (Eds.). Correspondence Art: Source Book for the Network of International Postal Art Activity. Contemporary Art Press, San Francisco.

Delgado, M. (1999). El animal público, Barcelona.

Ferrando García, P, Maral Martíb, J. (2016). Antes y después de Auschwitz La cinta Blanca/La cuestión Humana, Shangrila, Santander.

García Morales, L. \& Gutiérrez Colino, V. (2018). El arte de las cosas. ASRI. Arte y Sociedad. Revista de Investigación, (15), 137-149.

Gaspar, I. (2018). Hashtag (\#), el uso de nuevos métodos en las prácticas artísticas a partir de las dinámicas en las redes sociales. ASRI. Arte y Sociedad. Revista de Investigación, (15), 15-23.

Marcán Fiz, Simón, (1994). Del arte objetual al arte de concepto - 1960-1974 (6a Ed Akal), Madrid.

Martin, H. (1982). Should an eyelash last forever? An Interview with Ray Johnson. En: De Salvo, Donna (Ed.) (1999). Ray Johnson: correspondences. Catálogo de exposición. Ohio: Flammarion and Wexner Center for the Arts.

M, Martinez, J. (2000). Conceptos fundamentales de Arte. Alianza Editorial, Madrid.

Martinez Collado, Fco J. (1990). La paradoja del medio postal en el arte, Arte Postal, Facultad de Bellas Artes, Universidad de Castilla La Mancha, Cuenca.

Marcuse, H. (1978). La dimensión estética, Materiales, Barcelona.

Moray, M. (2016). El archivo audiovisual. Vicente, Pedro, (Dir.) Memoria y desacuerdo: políticas del archivo, registro y álbum familiar. Huesca. ORGANIZA: UIMP / Diputación Provincial de Huesca.

Torrecilla, E. (2018). El lenguaje de las interfaces en movimiento: La hibridación en la práctica artística de NoDOS (3). ASRI. Arte y Sociedad. Revista de Investigación, (15), 7-14. 